08

\title{
Импульсная электрическая прочность пленки полипропилена
}

\author{
(C) В.А. Закревский, ${ }^{1}$ В.А. Пахотин, ${ }^{1}$ Н.T. Сударь ${ }^{2,9}$ \\ ${ }^{1}$ Физико-технический институт им. А.Ф. Иоффре РАН, \\ 194021 Санкт-Петербург, Россия \\ ${ }^{2}$ Санкт-Петербургский политехнический университет Петра Великого, \\ 195251 Санкт-Петербург, Россия \\ ף e-mail: sudar53@mail.ru
}

(Поступило в Редакцию 25 мая 2016 г.)

Приведены результаты экспериментальных исследований электрической прочности пленки полипропилена толщиной $2 \mu \mathrm{m}$ при импульсном нагружении образцов в субмикросекундном и микросекундном диапазонах времен. Показано, что экспериментальные данные импульсного пробоя полимерной пленки можно описать, используя представления об ионизационном механизме пробоя полимеров, не связанном с развитием в них ударной ионизации молекул.

DOI: $10.21883 / J T F .2017 .02 .44133 .1907$

\section{Введение}

Изучение импульсной электрической прочности полимерных пленок представляет большой научный и практический интерес. Такие пленки используются в различных электротехнических устройствах в качестве изоляционных и диэлектрических слоев. В частности, они применяются в импульсных высоковольтных конденсаторах, что позволило существенно повысить рабочие характеристики этих устройств. Стремление к миниатюризации обусловливает необходимость использования в конденсаторах тонких полимерных пленок, толщины которых не превышают нескольких микрометров. В процессе эксплуатации конденсаторов пленки подвергаются воздействию электрических полей, напряженность которых может превышать $2-3 \mathrm{MV} / \mathrm{cm}$. Ясно, что в столь сильных электрических полях проблемы электрической прочности пленок приобретают первостепенное значение.

Вопросы импульсной электрической прочности различных полимерных пленок обсуждались в ряде работ. Электрические испытания проводились с использованием высоковольтных импульсов различной амплитуды, формы и длительности. Это либо короткие импульсы длительностью не более $10^{-7} \mathrm{~s}$ и протяженностью фронтов $\sim 1 \mathrm{~ns}[1-3]$, либо импульсы микросекундной (и более) длительности с существенно меньшей крутизной переднего фронта [4-6]. Установлено, что электрический пробой пленки происходит как на переднем фронте [4], так и на плато высоковольтных импульсов [1]. Причина наблюдаемого смещения момента пробоя с фронта на плато импульса напряжения в литературе не обсуждается. В ряде работ по импульсной электрической прочности полимеров отмечается уменьшение пробивной напряженности на импульсах по сравнению с величиной пробивной напряженности при медленно $(100-1000 \mathrm{~V} / \mathrm{s})$ возрастающем напряжении [5,7,8].
Целью настоящей работы было выяснение закономерностей импульсного электрического пробоя пленки полипропилена (ПП) и обсуждение возможных причин его развития (механизма электрического разрушения) в субмикросекундном диапазоне времен. Используя высокоскоростные методы регистрации с высоким временным разрешением, предполагалось выяснить условия возникновения пробоя на фронте и плато импульса.

\section{Методика эксперимента}

Объектом исследования служила двуосноориенированная пленка ПП толщиной $2 \mu \mathrm{m}$, которая закреплялась в специальной оправке между двумя стальными электродами и помещалась в конденсаторное масло. Один из электродов (плоский) находился под потенциалом земли. На второй (сферический электрод диаметром $2 \mathrm{~mm}$ ) подавался высоковольтный импульс отрицательной полярности. Осциллограмма импульса представлена на рис. 1. Используемый в работе генератор позволял формировать импульсы с протяженностью плато $\sim 7 \mu \mathrm{s}$.

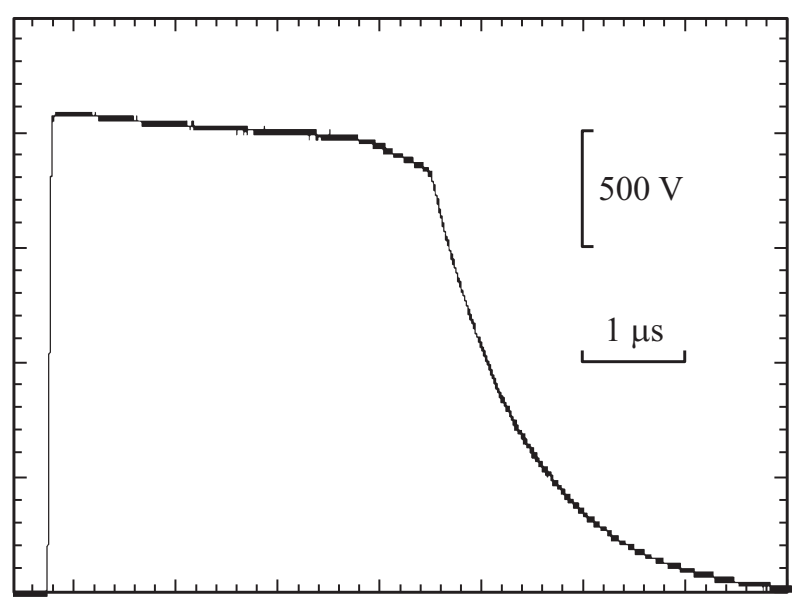

Рис. 1. Осциллограмма высоковольтного импульса. 
При изменении амплитуды импульса с 700 до $2000 \mathrm{~V}$ форма переднего фронта импульса была близка к линейной.

Вследствие особенностей конструкции генератора высоковольтных импульсов длительность переднего фронта импульса $t_{1}$ и его наклон изменялись с увеличением амплитуды импульса $V_{M}$, так что скорость подъема напряжения возрастала с $9.7 \mathrm{GV} / \mathrm{s}$ при $V_{M}=700 \mathrm{~V}$ до $19.4 \mathrm{GV} / \mathrm{s}$ при $V_{M}=2000 \mathrm{~V}$.

Испытание пленки на пробой заключалось в однократном воздействии на нее высоковольтного импульса. Амплитуда его была фиксирована для каждой серии испытаний образцов. В каждой серии испытывалось 30 образцов. Температура поддерживалась постоянной и равной $25^{\circ} \mathrm{C}$.

Для измерения напряжения на образце использовался широкополосный запоминающий осциллограф ADS2332 (интервал дискретизации $1 \mathrm{~ns}$ ) с высоковольтным широкополосным делителем TTHV250 с граничной частотой $300 \mathrm{MHz}$.

\section{Экспериентальные данные и их обсуждение}

На рис. 2 представлены типичные осциллограммы изменения напряжения на образце пленки, регистрируемые при пробое на плато $(a)$ и фронте $(b)$ импульса. Пробой пленки на осциллограммах регистрировался по

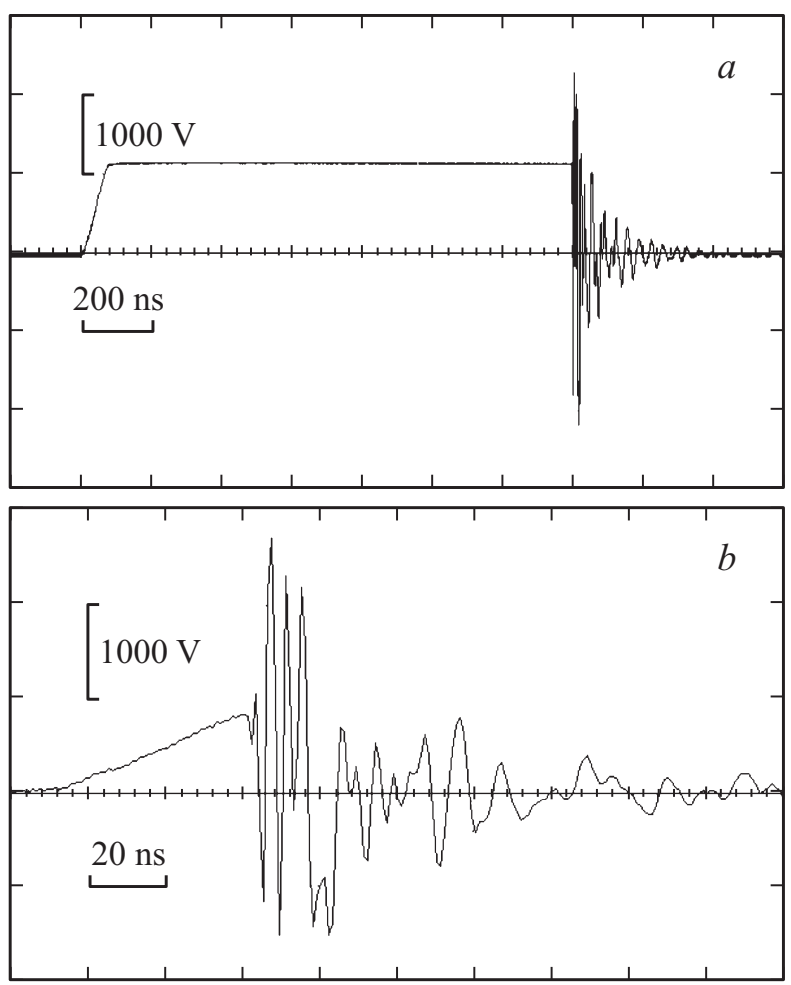

Рис. 2. Форма регистрируемых сигналов при пробое пленки на плато $(a)$ и фронте $(b)$ импульса.

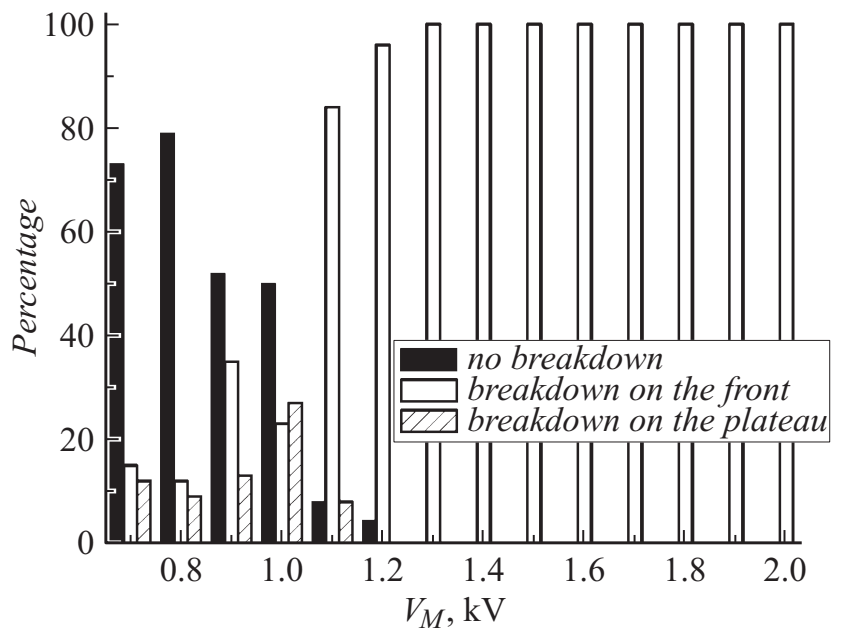

Рис. 3. Доли образцов в процентах по отношению к общему числу образцов в серии, соответствующие отсутствию пробоя образца, пробою на фронте и пробою на плато импульса при различных величинах амплитуды импульса.

резкому падению напряжения на образце и возникновению затухающих колебаний напряжения. В работе [9] было показано, что длительность пробоя пленки, т.е. время, в течение которого через пленку протекает ток пробоя плотностью $\sim 10^{7} \mathrm{~A} / \mathrm{cm}^{2}$, вызывающий разрушение диэлектрика в канале пробоя, не превышает $10^{-8}$ s. Регистрируемые затухающие (в течение $\sim 500 \mathrm{~ns}$ ) осцилляции напряжения представляют собой отклик измерительной схемы на кратковременный импульс тока.

Результаты опытов по разрушению (пробою) пленки ПП в электрическом поле при однократном воздействии на нее высоковольтного импульса представлены на рис. 3. На рисунке указаны доли образцов (в процентах) по отношению к общему числу образцов в серии, соответствующие отсутствию пробоя, пробою на фронте и пробою на плато импульса при различных значениях его амплитуды.

Видно, что при относительно невысоких значениях $V_{M}$ пробивалась только часть образцов, подвергнутых электрическим испытаниям, при этом вероятность пробоя на переднем фронте и на плато импульса была примерно одинаковой. Можно сказать, что при $V_{M}<1100 \mathrm{~V}$ значительная часть образцов „не успевает“ пробиться за время воздействия импульса с указанной амплитудой и длительностью. Если пробой наблюдался на плато импульса, то время до пробоя образца $t_{b r}$ могло изменяться в широких пределах от $t_{b r} \cong t_{1}$ до $t_{b r} \gg t_{1}$. При возрастании $V_{M}$ увеличивалась не только вероятность пробоя пленки, но и вероятность того, что пробой произойдет на фронте импульса. При $V_{M}>1200 \mathrm{~V}$ пробивались все образцы данной серии, причем пробой происходил только на переднем фронте импульса.

При проведении испытаний пленки наблюдается заметный разброс пробивных напряжений $V_{b r}$ и $t_{b r}$ для образцов одной серии, т. е. испытаний, выполненных при 
Значения амплитуды импульсов $V_{M}$, длительности их переднего фронта $t_{1}$, параметров распределения Вейбулла, используемого для описания распределения времен до пробоя, и рассчитанные на их основе математические ожидания времен пробоя $t_{e v}$ при различных $V_{M}$

\begin{tabular}{c|c|c|c|c|c|c|c|c|c}
\hline$V_{M}$ & 1100 & 1200 & 1300 & 1500 & 1600 & 1700 & 1800 & 1900 & 2000 \\
\hline$t_{1}, \mathrm{~ns}$ & 85 & 88 & 92 & 98 & 102 & 104 & 108 & 111 & 115 \\
\hline$t_{0}, \mathrm{~ns}$ & 63 & 62 & 47 & 46 & 55 & 55 & 56 & 50 & 50 \\
$m$ & 2.87 & 6.07 & 3.05 & 4.98 & 3.047 & 4.90 & 4.97 & 6.65 & 6.609 \\
$t_{e v}, \mathrm{~ns}$ & 56 & 58 & 42 & 42 & 50 & 50 & 52 & 47 & 46 \\
$r^{2}$ & 0.96 & 0.97 & 0.90 & 0.92 & 0.90 & 0.91 & 0.90 & 0.92 & 0.97
\end{tabular}

определенной величине $V_{M}$. Таким образом, величины $V_{b r}$ и $t_{b r}$ в каждой серии следует рассматривать как случайные величины, значения которых реализуются с определенной вероятностью.

Для описания распределения времен до пробоя использовалось двухпараметрическое распределение Вейбулла, которое в рассматриваемом случае имеет вид

$$
F\left(t_{b r}\right)=1-\exp \left[-\left(\frac{t_{b r}}{t_{0}}\right)^{m}\right],
$$

где $m$ - параметр формы, $t_{0}$ - параметр масштаба. Определив на основании экспериментальных данных параметры $m$ и $t_{0}$, можно рассчитать наиболее вероятное значение времени до пробоя (математическое ожидание) $t_{e v}$ при различных значениях $V_{M}[10]$. Результаты аппроксимации эмпирической функции распределения распределением Вейбулла представлены в таблице. Отметим, что достоверность аппроксимации $r^{2}$ результатов испытаний распределением Вейбулла превышает 0.9 для всех значений $V_{M}$, что свидетельствует об обоснованности его применения.

Переходя к обсуждению результатов экспериментов, прежде всего заметим, что в качестве возможной причины электрического пробоя полимерных диэлектриков при воздействии на них импульсного напряжения часто рассматривают ударную ионизацию полимерных макромолекул и образование электронных лавин $[1,2,8]$. Очевидно, что в условиях прыжкового транспорта носителей, характерного для органических материалов, возникновение электронных лавин в полимерах затруднено, поскольку длина свободного пробега в этих условиях не превышает $1 \mathrm{~nm}$, что соответствует межмолекулярному расстоянию, и, следовательно, энергия, набираемая электроном даже в поле $10^{7} \mathrm{~V} / \mathrm{cm}$, не превышает $1 \mathrm{eV}$, что существенно меньше энергии ионизации молекул. На это обстоятельство указывалось ранее в работах [11-13]. Критика представлений о лавинном характере пробоя полимеров для случая достаточно тонких пленок содержится также в [14], но аргументация авторов не выглядит достаточно убедительной, поскольку они не учитывают в своих рассуждениях прыжковый характер транспорта носителей.
В работах $[12,13]$ предложен новый, не связанный с ударной ионизацией, физический механизм, с единых позиций рассматривающий старение (ухудшение свойств полимеров в электрическом поле со временем) и пробой и объясняющий быстрое возрастание тока при электрическом пробое полимерных диэлектриков. Данный механизм базируется на представлении о полевой ионизации макромолекул. Накопление в полимерах электронов и дырок (положительных молекулярных ионов) приводит к возникновению дебаевского экранирования зарядов и, как следствие этого, снижению потенциала ионизации молекул, что, в свою очередь, ускоряет образование несвязанных зарядов в полимере. Таким образом, реализуется самоускоряющийся процесс, приводящий к возрастанию концентрации свободных носителей зарядов, что на определенном этапе приводит к взрывообразному изменению свойств полимера - потере им диэлектрических свойств, т.е. к его пробою вследствие протекания тока высокой плотности.

Вследствие кратковременности развития импульсного пробоя при его описании с позиции $[12,13]$ можно ограничиться лишь анализом наиболее быстропротекающего процесса, а именно рассмотрением полевой ионизации макромолекул при туннельном переходе электрона с уровня высшей занятой молекулярной орбитали макромолекулы (НОМО) на низшую свободную орбиталь (LUMO) другой молекулы. Отметим здесь, что такие (HOMO-LUMO) переходы определяют работу органических диодов Зинера [15].

В этом случае скорость накопления дырок (положительных молекулярных ионов) определяется уравнением

$$
\frac{d}{d t} p=k_{H L}\left(M_{0}-p\right)
$$

где $t-$ время, $M_{0}$ - начальная концентрация способных ионизоваться фрагментов макромолекул (мономерных звеньев), $p$ - концентрация дырок, $k_{H L}-$ константа скорости туннельной ионизации, обусловленной переходами HOMO-LUMO, определяемая как

$$
k_{H L}=v_{0} D_{H L}
$$

Здесь $v_{0}$ - частотный фактор, $D_{H L}-$ прозрачность барьера при переходе HOMO $\rightarrow$ LUMO.

Наиболее вероятно туннелирование вдоль направления поля, поэтому для оценки вероятности полевой ионизации молекул можно воспользоваться выражением

$$
D \cong \exp \left(-\frac{2 \sqrt{2 m}}{\hbar} \int_{x_{1}}^{x_{2}} \sqrt{|U(x)-E|} d x\right)
$$

где $E-$ энергия электрона, $U(x)$ - функция, задающая форму барьера, $\left(x_{2}-x_{1}\right)$ - ширина барьера, $m-$ масса электрона. 
Функция $U(x)-E$ определяется как

$$
U(x)-E=\Delta-\frac{e^{2}}{4 \pi \varepsilon_{0} \varepsilon x} \exp \left[-\frac{x}{r_{D}(p)}\right]-e F_{l o c} x,
$$

где $e-$ заряд электрона, $\varepsilon-$ диэлектрическая проницаемость полимера, $\varepsilon_{0}=8.85 \cdot 10^{-12} \mathrm{~F} / \mathrm{m}, r_{D}(p)-$ радиус Дебая, зависящий от концентрации зарядов в твердотельной плазме, $F_{l o c}$ - локальная напряженность электрического поля. Второе слагаемое в правой части уравнения (5) - кулоновский член, записанный с учетом эффекта экранирования. Высота потенциального барьера $\Delta$ при переходе HOMO $\rightarrow$ LUMO определяется шириной энергетической щели $E_{g}=\left|E_{\mathrm{HOMO}}-E_{\mathrm{LUMO}}\right|$. Здесь $E_{\mathrm{HOMO}}$ и $E_{\mathrm{LUMO}}-$ энергии уровней $\mathrm{HOMO}$ и LUMO. Учитывая эффект дебаевского экранирования зарядов, приводящий к снижению потенциала ионизации нейтральных молекул, высоту барьера $\Delta$ можно определить как $\Delta=E_{g}-\Delta I\left(r_{D}\right)$, где величина $\Delta I\left(r_{D}\right)$ [16] и дебаевский радиус экранирования $r_{D}(p)$ рассчитывались по формулам

$$
\begin{gathered}
\Delta I\left(r_{D}\right)=\frac{e^{2}}{4 \pi \varepsilon_{0} r_{D}(p)}, \\
r_{D}(p)=\sqrt{\frac{\varepsilon_{0} \varepsilon k_{B} T}{e^{2} p}},
\end{gathered}
$$

где $T$ - температура, $k_{B}-$ постоянная Больцмана.

Уравнение (2) решалось численно методом Гира дифференцирования назад с начальным условием $p=0$ при $t=0$. При этом полагалось, что $F_{l o c}=q V(t) / d(q-$ коэффициент электрических перенапряжений, $d-$ толщина образца). Напряжение на образце $V(t)$ в момент времени $\mathrm{t}$ определялось согласно соотношению

$$
V(t)= \begin{cases}\dot{V} t, & \text { при } \quad 0 \leq t \leq t_{1}, \\ V_{M}, & \text { при } t \geq t_{1},\end{cases}
$$

где $\dot{V}-$ скорость возрастания напряжения на переднем фронте импульса, определяемая как $\dot{V}=V_{M} / t_{1}$.

Все расчеты были выполнены для $T=298 \mathrm{~K}$ при $d=2 \mu \mathrm{m}$ и $\varepsilon=2.2$ [17]. При выборе значения $E_{g}$ мы основывались на данных работы [18], в которой эта величина для слабополярных полимерных диэлектриков полагается равной 6-7eV. Поэтому для ПП принималось $E_{g}=7 \mathrm{eV}$. Принималось также, что $M_{0}=10^{21} \mathrm{~cm}^{-3}$.

На рис. 4 представлены зависимости $p(t) / M_{0}$, характеризующие увеличение концентрации зарядов в полимере при воздействии на пленку импульсного напряжения, изменяющегося со временем согласно (8). Зависимости $p(t) / M_{0}$ на рис. 4, $a$ соответствуют расчетам, выполненным при различных значениях $V_{M} \geq 1200 \mathrm{~V}$, когда $V(t)=\dot{V} t$, с учетом снижения потенциала ионизации макромолекул вследствие эффекта дебаевского экранирования (кривые $1-3)$ и без его учета (кривые $\left.1^{\prime}-3^{\prime}\right)$.

Видно, что кривые 1-3 при достижении некоторого момента времени, зависящего от величины $V_{M}$, становятся практически вертикальными. К этому моменту
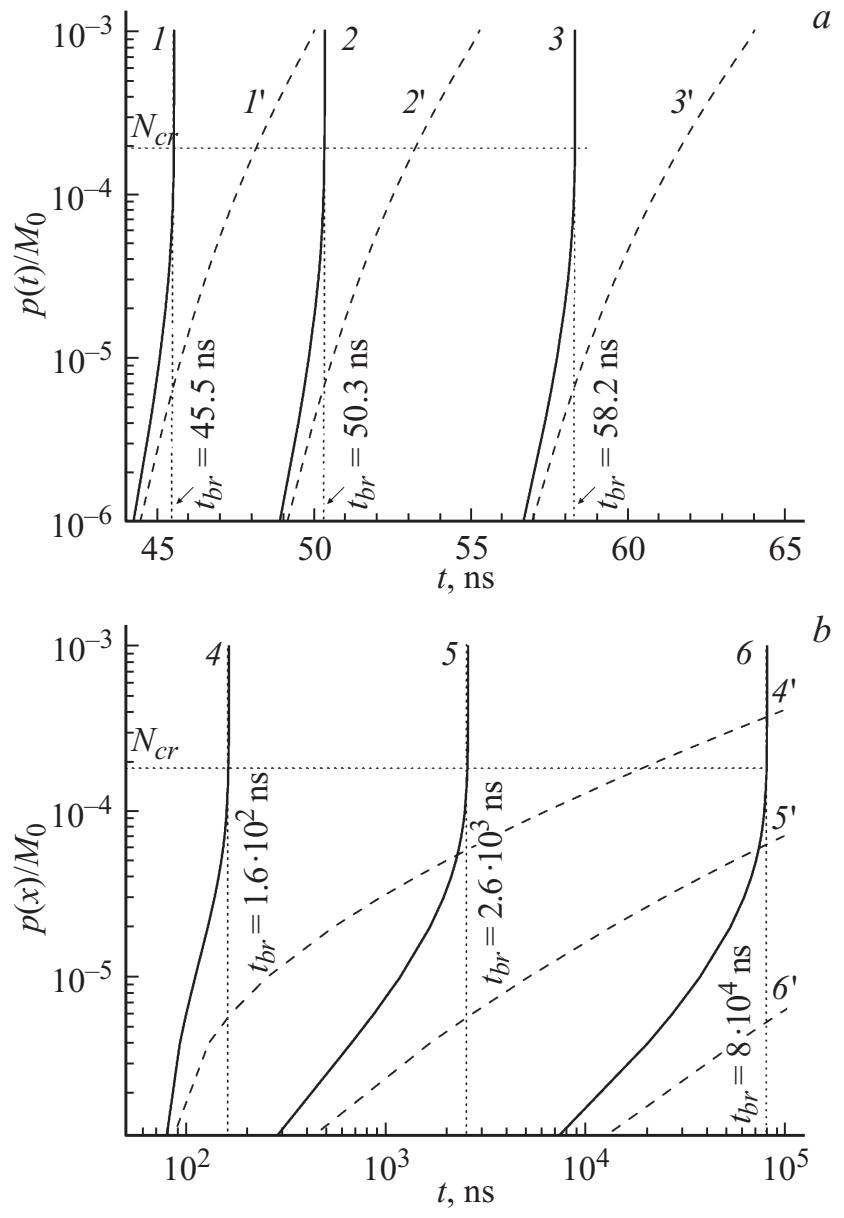

Рис. 4. Увеличение концентрации зарядов в пленке со временем при воздействии импульсного напряжения различной амплитуды $V_{M}$. Сплошные линии - расчет выполнен при $\Delta=E_{g}-\Delta I(p)$, пунктирные линии - расчет выполнен при $\Delta=E_{g}=\operatorname{const}(p) ; a-1$ и $1^{\prime}-2000 \mathrm{~V}, 2$ и $2^{\prime}-1600 \mathrm{~V}$, 3 и $3^{\prime}-1200 \mathrm{~V}, b-4$ и $4^{\prime}-750 \mathrm{~V}, 5$ и $5^{\prime}-725 \mathrm{~V}, 6$ и $6^{\prime}-700 \mathrm{~V}$.

времени концентрация зарядов в пленке достигает критического значения $N_{\mathrm{cr}} \approx 2 \cdot 10^{17} \mathrm{~cm}^{-3}$ и вследствие самоускоряющегося процесса полевой ионизации молекул происходит взрывообразное возрастание концентрации носителей зарядов. В результате наблюдается резкий рост тока, вызывающего пробой диэлектрика, что согласуется с результатами экспериментов по пробою полимеров [19]. Данный момент времени в дальнейшем рассматривался нами как момент пробоя, а время от начала возрастания напряжения на образце $(t=0)$ до момента пробоя обозначалось как $t_{b r}$. Как отмечалось выше, при $V_{M} \geq 1200 \mathrm{~V}$ в экспериментах пробой всегда наблюдался на фронте импульса. Поскольку полученные на основании данных рис. $4, a$ расчетные значения $t_{b r}<t_{1}$, то зависимости $p(t) / M_{0}$ соответствуют ситуации, когда пробой также происходит на фронте импульса. В этом случае быстрое возрастание концентрации зарядов в пленке обусловлено как возрастанием со временем напряженно- 
сти электрического поля, так и увеличением $\Delta I\left(r_{D}\right)$. При $q=2.7$ наблюдается наилучшее согласие расчетных и экспериментальных значений $t_{b r}$. Для $V_{M}$, равных 1200 , 1600 и $2000 \mathrm{~V}$, расчетные значения $t_{b r}$ равны соответственно 58.2, 50.3 и $45.5 \mathrm{~ns}$. Эти результаты хорошо согласуются с величинами математического ожидания времени пробоя $t_{e v}$, представленными в таблице.

Смещение $t_{b r}$ с фронта на плато импульса происходит при таких значениях $V_{M}$, когда за время $t_{1}$ концентрация зарядов $p$ не успевает достичь критического значения. При $t>t_{1}$ накопление зарядов протекает уже в условиях постоянной (не изменяющейся со временем) напряженности электрического поля, равной $F_{l o c}=q V_{M} / d$. Поэтому увеличение скорости накопления зарядов со временем вплоть до момента пробоя будет определяться только скоростью увеличения $\Delta I\left(r_{D}\right)$. В качестве характеристики электрической прочности в этом случае следует использовать величину $t_{b r}=t_{1}+t_{2}$, где $t_{2}-$ время действия на полимер постоянного напряжения $V_{M}$. На рис. $4, b$ представлена зависимость $p(t) / M_{0}$, соответствующая ситуации, когда пробой должен происходить на плато импульса. Видно, что при уменьшении амплитуды импульса с 750 до $700 \mathrm{~V}$ время до пробоя возрастает в $\sim 500$ раз с $1.6 \cdot 10^{2}$ до $8 \cdot 10^{4} \mathrm{~ns}$.

Отметим, что расчеты $p(t) / M_{0}$, выполненные без учета снижения высоты потенциального барьера $\Delta$ (кривые $1^{\prime}-3^{\prime}$ и $4^{\prime}-6^{\prime}$ на рис. 4$)$, показывают, что значения $p(t) / M_{0}$ плавно возрастают со временем без резкого ускорения. Этот результат позволяет сделать важный для физики пробоя полимеров вывод о том, что результаты расчетов, выполненных без учета „дебаевского“ снижения высоты потенциального барьера, не согласуются с результатами экспериментов.

Таким образом, полученные при импульсном нагружении данные вполне определенно указывают на четкую зависимость времени жизни полимерной пленки в электрическом поле от его напряженности. Этот вывод находится в согласии с результатами изучения долговечности полимеров в постоянном электрическом поле $[13,19-21]$. В обоих случаях (как в постоянном поле, так и в поле, напряженность которого возрастает со временем) долговечность определяется скоростью накопления повреждений макромолекул, т.е. скоростью их ионизации.

\section{Заключение}

Таким образом, в настоящей работе получены данные об электропрочностных свойствах тонкой пленки ПП при импульсном нагружении образцов в субмикросекундном и микросекундном диапазонах времен.

Показано, что экспериментальные данные импульсного пробоя полимерной пленки можно описать, используя представления об ионизационном механизме пробоя полимеров, не связанном с развитием в них ударной ионизации.
На основании полученных в настоящей работе данных следует, что электрическое разрушение полимеров при импульсном нагружении состоит в накоплении дефектов макромолекул, заканчивающимся при достижении их критической концентрации резким увеличением протекающего через полимер тока, т.е. пробоем. Поэтому электрические свойства полимерных пленок при импульсном нагружении (с рассматриваемыми временнб́ми характеристиками импульсов) следует характеризовать так же, как и в постоянном поле, пробивной напряженностью и временем жизни (долговечностью), зависящих от таких параметров импульса, как крутизна его фронта и амплитуда.

\section{Список литературы}

[1] Kitani I., Arii K. // IEEE Trans. Elect. Insul. 1982. Vol. EI-17. № 3. P. 228-233.

[2] Kitani I., Arii K. // Jpn. J. Appl. Phys. 1983. Vol. 22. N 5R. P. 857-862.

[3] Wang J., Zhou Y.X., Yan P., Huang W.L., Li G.J., Zhang S.C., Sun G.S. // J.Electrostatics. 2005. Vol. 63. N 6-10. P. 665-671.

[4] Suzuoki Y., Hattori K., Mizutani T.,Yoshifuji N. // IEEE 5th Intern. Conf. Conduct. Breakdown in Sol. Dielectrics. 1995. P. 641-645.

[5] Watson D.B. // J. Phys. D. Appl. Phys. 1972. Vol. 5. P. 410-414.

[6] Watson D.B. // Phys. D. Appl. Phys. 1996. Vol. 29. P. 3129-3131.

[7] Электрические свойства полимеров / Под ред. Б.И. Сажина. Л.: Химия, 1986. 224 с.

[8] Artbauer J., Griač J. // Proc. IEEE. 1965. Vol. 112. N 4. P. 818-818.

[9] Пахотин В.А., Закревский В.А., Сударь Н.Т. // ЖТФ. 2015. Т. 85. Вып. 8. С. 40-45.

[10] Капур К., Ламберсон Л. Надежность и проектирование систем. М.: Мир, 1980. 604 с.

[11] Закревский В.А., Сударь Н.T. // ФТТ. 2005. Т. 47. Вып. 5. C. 931-936.

[12] Закревский В.А., Сударь Н.T. // ФТТ. 2013. Т. 55. Вып. 7. C. $1298-1303$.

[13] Zakrevskii V.A., Pakhotin V.A., Sudar N.T. // J. Appl. Phys. 2014. Vol. 115. P. 234101.

[14] Dissado L.A., Fothergill J.C. Electrical Degradation and Breakdown in Polymers. London: Peter Peregrinus, 1992.

[15] Kleemann H., Gutierrez R., Lindner F., Avdoshenko S., Pedro D. Manrique P.D., Lüssem B., Cuniberti G., Leo K. // Nano Lett. 2010. Vol. 10. P. 4929-4934.

[16] Griem H.R. // Phys. Rev. 1962. Vol. 128. N 3. P. 997-1003.

[17] Физические величины. Справочник / Под ред. И.С. Григорьева, Е.3. Михайлова. М.: Энергоатомиздат, 1991. 1232 с.

[18] Вилесов Ф.И., Загрубский А.А., Сухов Д.А. // ФТТ. 1969. Вып. 11. С. 3409-3410.

[19] Hikita M., Kanno I., Sawa G., Ieda M. // Jpn. J. Appl. Phys. 1985. Vol. 24. N 8. P. 984-987.

[20] Liufu D., Kao K.C. // J. Appl. Phys. 1999. Vol. 85. N 2. P. 1089-1094.

[21] Zakrevskii V.A., Sudar N.T., Zaopo A., Dubitsky Yu.A. // J. Appl. Phys. 2003. Vol. 93. N 4. P. 2135-2139. 